\title{
Primary Antiphospholipid Syndrome with and Without Acute Myocardial Infarction/ Angina: A Cross-Sectional Study
}

\author{
Jozélio Freire de Carvalho (D) · Carlos Ewerton Maia Rodrigues (D)
}

Received: November 10, 2021 / Accepted: December 14, 2021 / Published online: January 9, 2022

(c) The Author(s) 2022

\section{ABSTRACT}

Introduction: Acute myocardial infarct/angina (AMI-A) is a possible complication in primary antiphospholipid syndrome (pAPS) patients. This study compares data obtained from pAPS patients with and without AMI-A.

Methods: This cross-sectional study of 66 (85.2\% female) pAPS patients (Sidney criteria). Demographics, clinical data, medication use, and antiphospholipid antibodies were evaluated. Patients were divided into two groups: pAPS with AMI-A and pAPS without AMI-A.

Results: Sixty-six patients with primary APS (six with AMI-A and 60 without AMI-A) were selected. They were similar for demographics, disease duration, and anthropometrics $(p>0.05)$. Patients with AMI-A compared to those patients without AMI-A had more frequently dyslipidemia (66 vs. $28 \%, p=0.05$ ), systemic hypertension ( 83 vs. $37 \%, p=0.02$ ),

J. F. de Carvalho ( $\square)$

Institute for Health Sciences From Federal

University of Bahia, Rua das Violetas, 42, ap. 502,

Pituba, Salvador, BA, Brazil

e-mail: jotafc@gmail.com

C. E. M. Rodrigues

Medical Sciences, Medical School, University of

Fortaleza (Unifor), Fortaleza, Brazil

C. E. M. Rodrigues

Department of Internal Medicine, Federal

University of Ceará, Fortaleza, Brazil and increased levels of lipoprotein (a) (116 \pm 67 vs. $36 \pm 35 \mathrm{mg} / \mathrm{dl}, \quad p=0.0002)$. Interesting, current physical activity ( 66.7 vs. $23 \%, p=0.04$ ) was more seen in the first group when compared to the second one. Patients with AMI-A used more statins (66 vs. $22 \%, p=0.017$ ) and acetylsalicylic (100 vs. $28 \%, p=0.05$ ). Higher median levels of IgM anticardiolipin antibodies [70 (0-120) vs. $9 \quad(0-120), p=0.03]$ were observed in the first group.

Conclusions: pAPS patients and AMI-A have distinct clinical and laboratory spectra from those without AMI-A. It is characterized by dyslipidemia and hypertension, hyper lipoprotein(a), and a lower IgM anticardiolipin frequency.

Keywords: Antiphospholipid syndrome; Hughes syndrome; Acute myocardial infarction; Angina; Cardiovascular diseases 


\section{Key Summary Points}

APS is associated with higher rates of cardiovascular disease.

About $2.8 \%$ of patients with APS may develop into acute myocardial infarction (MAI).

The present study compared APS patients with and without MAI-angina; the first group showed a higher frequency of dyslipidemia, hypertension, hyper lipoprotein(a), and a lower IgM anticardiolipin frequency.

\section{INTRODUCTION}

Antiphospholipid syndrome (APS) is an acquired thrombophilia characterized by recurrent thrombosis and/or obstetric events with or without thrombocytopenia in the presence of antiphospholipid antibodies [1].

APS is linked to higher rates of cardiovascular disease (CVD) [2]. This fact comes from traditional cardiovascular risk factors, but the contributions of inflammatory and autoimmune processes have been increasingly recognized $[3,4]$. The most common cardiac manifestations include valvopathies and coronary artery disease in primary APS (15\%) and APS associated with autoimmune diseases (30\%). In addition, about $2.8 \%$ of patients with APS may develop into myocardial infarction partially due to accelerated atherosclerosis or coronary embolism, especially in young people [2]. In this regard, Sacré et al. [5] showed that occult myocardial ischemia's prevalence was seven times higher in the patients with APS when compared to the controls. However, no study that systematically evaluated APS patients with acute myocardial infarction-angina (AMI-A) and those without this clinical picture was performed yet.

Therefore, this study aims to assess the possible association of clinical, laboratory, and treatment features among patients with primary APS (pAPS), with and without associated AMI-A.

\section{METHODS}

\section{Subjects}

This cross-sectional study included 66 patients regularly followed at our private clinic. All patients fulfilled the Sidney criteria for primary APS [3]. Patients of both genders with ages equal to or greater than 18 years were included. Patients with another systemic autoimmune disease such as rheumatoid arthritis or systemic lupus erythematosus were excluded from the study. The authors fulfilled the Helsinki World Declaration, and all participants signed informed consent to participate in this study, and the Local Ethical Committee approved it in March 2020.

\section{Clinical Features}

All patients had their medical histories and demographic data collected through review of their medical charts to evaluate current or previous arterial thrombosis events, documented strokes, transient ischemic attacks, peripheral artery occlusions, angina or AMI, venous thrombosis (documented deep vein thrombosis and/or pulmonary embolisms), thrombocytopenia $\left(100,000\right.$ platelets $/ \mathrm{mm}^{3}$ on at least two distinct occasions), disease duration, comorbidities (including systemic arterial hypertension and diabetes), height, weight, body mass index, smoking, sedentary lifestyle, and medications. Stroke and vascular events were confirmed by imaging such as computed tomography (CT), magnetic resonance imaging (MRI), computed tomography angiography (CTA), and/or magnetic resonance angiography (MRA). Other vascular events, arterial (including limb ischemia) or venous, were diagnosed using imaging, Doppler sonography, ventilation/perfusion scintigraphy, and/or arteriography. Thrombocytopenia was defined as a platelet count of less than $100,000 / \mathrm{mm}^{3}$ on two consecutive separated occasions. Livedo 
reticularis diagnosis was based on typical livedo reticularis on the physical examination. In the case of clinical doubt, a skin biopsy was performed. The presence of comorbidities such as smoking, systemic arterial hypertension (blood pressure $>140 / 90 \mathrm{mmHg}$ or anti-hypertensive drug use), and dyslipidemia (defined by the National Cholesterol Education Program [6]), as well all medication used (e.g., glucocorticoid, warfarin, hydroxychloroquine, statins, and acetylsalicylic acid), were also recorded. Individuals engaging in less than 150 min of moderate-intensity exercise per week were considered sedentary lifestyles [6]. Thyroid dysfunction was defined as hypothyroidism (low free T4 or high TSH levels) and hyperthyroidism (high free T4 or low TSH levels), independently of clinical thyroid symptoms. Lipoprotein (a) was measured using immunoturbidimetry (DiaSorin, Sallugia, Italy). Normal values were those lower than $30 \mathrm{mg} / \mathrm{dl}$.

AMI was defined by the presence of signs or symptoms compatible with ischemia, associated with the presence of ECG or other imaging abnormalities interpreted as ischemia, and a rising and/or falling pattern of biomarkers (troponin and/or CK-MB) over at least $6 \mathrm{~h}$, with at least one value above twice the normal upper limit for the assay, used [4]. Angina was defined as $\geq 2$ episodes of typical angina (thoracic pain). Angina was classified according to one of the four following categories [7]: (1) typical angina (fulfilling all the following three criteria (a) retrosternal chest discomfort, (b) chest discomfort precipitated by exertion, and (c) prompt relief [the $30 \mathrm{~s}-10 \mathrm{~min}$ ] of chest discomfort by rest or nitroglycerin) (2) atypical angina (two out of three criteria for typical angina pectoris), (3) non-anginal chest discomfort (one out of three criteria for typical angina), and (4) other chest discomforts (none of the above criteria for typical angina pectoris).

\section{Antiphospholipid Antibodies}

IgG and IgM anticardiolipin antibodies were determined using enzyme-linked immunosorbent assay (ELISA). These antibody levels were expressed in GPL or MPL units (Louisville, KY,
USA). The normal range was $<40$ GPL or MPL. In addition, lupus anticoagulant was evaluated using a functional test and was performed as described by the International Society for Haemostasis and Thrombosis.

\section{Statistical Analysis}

Results were expressed as mean \pm standard deviation (SD). Statistical analysis was carried out using JASP 0.12.2 version Software (Amsterdam, The Netherlands). Student's $t$ test analyzed the data to compare means, and comparisons between proportions were calculated using the Fisher's exact test. $P$ values below 0.05 were considered statistically significant.

\section{RESULTS}

Sixty-six patients with primary APS (six with AMI-A and 60 without AMI-A) were included. The groups (pAPS with AMI-A and pPAPS without AMI-A) were similar regarding age, gender, Caucasian race, disease duration, and anthropometrics $(p>0.05)$ (Table 1). Four out of six $(66.7 \%)$ patients had AMI (non-Q infarction) and were treated with angioplasty with stent insertion; $3 / 6(50 \%)$ had angina, all of them had typical angina, and 1/6 (16.7\%) had simultaneous angina and AMI, treated with angioplasty with stent.

Patients with AMI-A compared to those patients without AMI-A had more frequently dyslipidemia (66 vs. $28 \%, p=0.05$ ), systemic hypertension ( 83 vs. $37 \%, p=0.02)$, and increased levels of lipoprotein (a) (116 \pm 67 vs. $36 \pm 35 \mathrm{mg} / \mathrm{dl}, p=0.0002$ ). Interestingly, current physical activity (66.7 vs. $23 \%, p=0.04$ ) was frequent in the first group compared to the second one. The other APS manifestations and comorbidities did not differ significantly (Table 2). Renal thrombotic microangiopathy was seen in 5/66 pAPS. All five had systemic hypertension during TMA, and $2 / 5$ had no more hypertension during this study.

Patients with AMI-A used more statins (66 vs. $22 \%, p=0.017)$ and acetylsalicylic (100 vs. $28 \%, p=0.05)$. No other significant differences were detected (Table 3 ). 
Table 1 Comparison of demographic and anthropometric data and disease duration in patients with primary antiphospholipid syndrome (pAPS) with and without acute myocardial infarction (AMI-A)

\begin{tabular}{llll}
\hline & $\begin{array}{l}\text { pAPS with AMI-A } \\
\mathbf{n = 6}\end{array}$ & $\begin{array}{l}\text { pAPS without AMI-A } \\
\boldsymbol{n}=\mathbf{6 0}\end{array}$ & $\boldsymbol{p}$ \\
\hline Mean age (years) & $44.4 \pm 16.4$ & $38.6 \pm 10.4$ & 0.25 \\
Female sex, $n$ (\%) & $5(83)$ & $50(83)$ & 1.00 \\
White race, $n$ (\%) & $4(67)$ & $46(81)$ & 0.59 \\
Weight $(\mathrm{kg})$ & $75.7 \pm 16.7$ & $73.3 \pm 19.7$ & 0.77 \\
Height $(\mathrm{cm})$ & $164 \pm 7.6$ & $160.9 \pm 8.3$ & 0.39 \\
Body mass index $\left(\mathrm{kg} / \mathrm{m}^{2}\right)$ & $27.9 \pm 5.6$ & $28.1 \pm 6.9$ & 0.97 \\
Disease duration (months) & $45.7 \pm 38.4$ & $73.1 \pm 62.0$ & 0.51 \\
\hline
\end{tabular}

Data are expressed as mean \pm standard deviation or as percentage

Table 2 Clinical data, cardiovascular events, comorbidities, and lifestyles of patients with primary antiphospholipid syndrome (pAPS) with and without acute myocardial infarction (AMI-A)

\begin{tabular}{llll}
\hline & $\begin{array}{l}\text { pAPS with AMI-A } \\
\mathbf{n}=\mathbf{6}\end{array}$ & $\begin{array}{l}\text { pAPS without AMI-A } \\
\boldsymbol{n}=\mathbf{6 0}\end{array}$ & $\boldsymbol{p}$ \\
\hline Arterial events, $n$ (\%) & $5(83)$ & $33(55)$ & 0.19 \\
Stroke, $n$ (\%) & $3(50)$ & $22(37)$ & 0.53 \\
Sneddon, $n$ (\%) & $1(17)$ & $12(20)$ & 0.90 \\
Venous events, $n$ (\%) & $4(67)$ & $28(47)$ & 0.36 \\
Deep venous thrombosis, $n$ (\%) & $3(50)$ & $39(60)$ & 0.47 \\
Pulmonary thromboembolism, $n$ (\%) & $2(33)$ & $13(22)$ & 0.52 \\
Thrombocytopenia, $n$ (\%) & 0 & $7(12)$ & 0.43 \\
Arterial systemic hypertension, $n$ (\%) & $5(83)$ & $22(37)$ & $\mathbf{0 . 0 2}$ \\
Dyslipidemia, $n$ (\%) & $4(66.7)$ & $17(28)$ & $\mathbf{0 . 0 4}$ \\
Family history of CAD & 0 & $7(12)$ & 0.37 \\
Current smoking, $n$ (\%) & 0 & $14(23)$ & 0.36 \\
Previous smoking, $n$ (\%) & $2(33)$ & $14(23)$ & 0.61 \\
Thyroidopathy, $n$ (\%) & 0 & $9(15)$ & 0.35 \\
Current physical activity, $n(\%)$ & $14(23)$ & $\mathbf{0 . 0 4}$ \\
Obstetric events, $n$ (\%) & $4(66)$ & $19(32)$ & 1.00 \\
Seizures, $n$ (\%) & $2(33)$ & $7(12)$ & 0.38 \\
Lipoprotein (a) & 0 & $36 \pm 35$ & $\mathbf{0 . 0 0 0 2}$ \\
\hline
\end{tabular}


Table 3 Drug use in patients with primary antiphospholipid syndrome (pAPS) with and without acute myocardial infarct/ angina

\begin{tabular}{llll}
\hline & $\begin{array}{l}\text { pAPS with AMI-A } \\
\boldsymbol{n}=\mathbf{6}\end{array}$ & $\begin{array}{l}\text { pAPS without AMI-A } \\
\boldsymbol{n}=\mathbf{6 0}\end{array}$ & $\boldsymbol{p}$ \\
\hline Current glucocorticosteroid use, $n$ (\%) & 0 & $6(10)$ & 0.42 \\
Previous glucocorticosteroid use, $n(\%)$ & $1(17)$ & $25(42)$ & 0.18 \\
Current warfarin use, $n$ (\%) & $6(100)$ & $47(78)$ & 0.082 \\
Current hydroxychloroquine use, $n$ (\%) & $5(83)$ & $6(100)$ & 0.65 \\
Current statins use, $n$ (\%) & $4(66.7)$ & $13(22)$ & $\mathbf{0 . 0 1 7}$ \\
Current acetylsalicylic acid use, $n(\%)$ & $6(100)$ & $17(28)$ & $\mathbf{0 . 0 5}$ \\
\hline
\end{tabular}

$A M I-A$ acute myocardial infarct /angina

Table 4 Antiphospholipid and antinuclear antibodies are present in patients with primary antiphospholipid syndrome (pAPS) with and without acute myocardial infarct /angina (AMI-A)

\begin{tabular}{llll} 
& $\begin{array}{l}\text { pAPS with AMI-A } \\
\mathbf{n = 6}\end{array}$ & $\begin{array}{l}\text { pAPS without AMI-A } \\
\boldsymbol{n}=\mathbf{6 0}\end{array}$ & $\boldsymbol{p}$ \\
\hline Lupus anticoagulant positivity, $n(\%)$ & $3(50)$ & $30(50)$ & 1.00 \\
IgG aCL positivity, $n$ (\%) & $4(67)$ & $30(50)$ & 0.28 \\
IgM aCL positivity, $n$ (\%) & $2(33)$ & $30(58)$ & 0.12 \\
IgG or IgM aCL positivity, $n(\%)$ & $5(83)$ & $39(60)$ & 0.91 \\
IgM anticardiolipin, MPL & $70(0-120)$ & $9(0-120)$ & $\mathbf{0 . 0 3}$ \\
Antinuclear antibodies positivity, $n(\%)$ & $2(33)$ & $18(30)$ & 0.52 \\
\hline
\end{tabular}

$A M I-A$ acute myocardial infarct/angina, $a C L$ anticardiolipin antibodies, $I g$ immunoglobulin

Autoantibody analysis in patients with pAPS (with AMI-A and those without AMI-A) groups is shown in Table 4. Higher median levels of IgM anticardiolipin [70 (0-120) vs. 9 (0-120), $p=0.03]$ were observed in the first group. No other significant differences were detected.

\section{DISCUSSION}

This study determined that patients suffering from pAPS associated with AMI-A are more related to dyslipidemia and systemic hypertension, besides hyperlipoproteinemia (a).

The strengths of this study are: first, the inclusion of only patients who fulfilled the international criteria for APS; second, the exclusive inclusion of primary APS. It is well known that lupus, rheumatoid arthritis, and several other rheumatic diseases themselves may be associated with other factors that might hamper data understanding, such as therapy (glucocorticoids) and accelerated atheromatous [8].

Traditional risk factors for AMI-A are well known in rheumatic diseases and APS $[9,10]$. The current study could confirm this strong association between dyslipidemia and systemic hypertension. In this regard, lipoprotein (a) is an underlying risk factor for cardiovascular disease, myocardial infarction, atherosclerosis process, and aortic valve stenosis. [11]. In APS, hyper-lipoprotein (a) is also linked to atherosclerosis [10, 12]. A very significant 
difference was seen in patients with AMI-A, confirming the previous data in the present study. Furthermore, it is known that renal microangiopathy may cause systemic hypertension. This alteration was seen in $7.5 \%$ of the pAPS of our patients.

Notably, a positive association was found with a higher frequency of current physical activity. For many years, it was suggested to avoid physical exercise after a cardiovascular event; currently, it is a consensus that exercise training ought to be part of cardiac therapy programs [13]. Therefore, we have seen subjects after AMI-A doing exercise.

Statins and acetylsalicylic acid are universally used and recommended to patients with myocardial infarction [14], and we also saw the use of these two drugs in our patients with AMI.

The lower frequency of IgM anticardiolipin positivity observed in our patients with AMI may represent the general picture that lupus anticoagulant antibody is more linked to arterial events. Interestingly, in a study with $50 \mathrm{AMI}$ compared to 30 controls, lupus anticoagulant was $59.2 \mathrm{U} / \mathrm{ml}$, IgM $1.14 \mathrm{U} / \mathrm{ml}$, and IgG anticardiolipin was $1.26 \mathrm{U} / \mathrm{ml}$, respectively [15]. This fact aligns with the lower frequency of IgM anticardiolipin observed in our patients. Furthermore, a study involving 203 patients showed that the chance for myocardial infarction was 5.3 (95\% CI 1.4-20.8) in patients with lupus anticoagulant [16].

Our study has some limitations: (1) the relatively low number of participants. Even though all patients are presenting pAPS, it would be exciting to have many other patients with this coagulopathy; (2) it is a unicentric observation, and it would be desired to have a multicentric with multiethnic participation in order to confirm the present data; (3) the causal effect (especially concerning treatment) could not be assessed due to the cross-sectional study design; and (4) the absence of a healthy control group matched by sex and age. Therefore, future studies with many APS participants, especially with multicentric cohorts with AMI-A, are expected to ensure the current data.

\section{CONCLUSIONS}

In conclusion, this study has shown that individuals with pAPS associated with AMI-A have more traditional risk factors for cardiovascular diseases, such as dyslipidemia and hypertension, besides hyper-lipoprotein (a) and lower levels of IgM anticardiolipin antibodies. Therefore, physicians who deal with pAPS patients should pay attention to traditional risk factors and non-traditional and antiphospholipid antibodies.

\section{ACKNOWLEDGEMENTS}

The authors would like to thank Sergio Ribeiro for the English revision of the manuscript, and no funding was given to him. Data sharing does not apply to this article, as no datasets were generated or analyzed during the current study. Finally, we thank the participants of this study.

Funding. No funding or sponsorship was received for this study or publication of this article.

Authorship. All named authors meet the International Committee of Medical Journal Editors (ICMJE) criteria for authorship for this article, take responsibility for the integrity of the work as a whole, and give their approval for this version to be published.

Author Contributions. JFC: design, data collection, writing, data analysis, statistical analysis, submission. CEMR: data analysis, writing, revision.

Disclosures. JF Carvalho has no conflicts of interest. CEM Rodrigues has nothing to disclose.

Compliance with Ethics Guidelines. The authors declare that the World Medical Association Declaration of Helsinki was followed. 
Informed consent was obtained from all patients for publication of this study. Federal University of Bahia's ethical committee approved this study.

Open Access. This article is licensed under a Creative Commons Attribution-NonCommercial 4.0 International License, which permits any non-commercial use, sharing, adaptation, distribution and reproduction in any medium or format, as long as you give appropriate credit to the original author(s) and the source, provide a link to the Creative Commons licence, and indicate if changes were made. The images or other third party material in this article are included in the article's Creative Commons licence, unless indicated otherwise in a credit line to the material. If material is not included in the article's Creative Commons licence and your intended use is not permitted by statutory regulation or exceeds the permitted use, you will need to obtain permission directly from the copyright holder. To view a copy of this licence, visit http://creativecommons.org/licenses/by$\mathrm{nc} / 4.0 /$.

\section{REFERENCES}

1. Hughes GR. Thrombosis, abortion, cerebral disease, and the lupus anticoagulant. Br Med J (Clin Res Ed). 1983;287:1088-9.

2. Kolitz T, Shiber S, Sharabi I, et al. Cardiac manifestations of antiphospholipid syndrome with focus on its primary form. Front Immunol. 2019;10:941.

3. Miyakis S, Lockshin MD, Atsumi T, Branch DW, Brey RL, Cervera R, et al. International consensus statement on an update of the classification criteria for definite antiphospholipid syndrome (APS). J Thromb Haemost. 2006;4(2):295-306.

4. Safford MM, Parmar G, Barasch CS, et al. Hospital laboratory reporting may be a barrier to detection of 'microsize' myocardial infarction in the US: an observational study. BMC Health Serv Res. 2013;13: 162.

5. Sacré K, Brihaye B, Hyafil F, et al. Asymptomatic myocardial ischemic disease in antiphospholipid syndrome. Arthritis Rheum. 2010;62:2093-100.
6. Expert Panel on Detection, Evaluation, and Treatment of High Blood Cholesterol in Adults. Executive Summary of the Third Report of the National Cholesterol Education Program (NCEP) Expert Panel on Detection, Evaluation, and Treatment of High Blood Cholesterol in Adults (Adult Treatment Panel III). JAMA 2001;285:2486-97.

7. Diamond GA. A clinically relevant classification of chest discomfort. J Am Coll Cardiol. 1983;1(2 Pt 1):574-5. https://doi.org/10.1016/S0735-1097(83)80093-X.

8. Schier O, Tosevski C, Glazier RH, Hogg-Johnson S, Badley EM. Incident myocardial infarction associated with major types of arthritis in the general population: a systematic review and meta-analysis. Ann Rheum Dis. 2017;76(8):1396-404. https://doi. org/10.1136/annrheumdis-2016-210275.

9. da Silva FF, Levy RA, de Carvalho JF. Cardiovascular risk factors in the antiphospholipid syndrome. J Immunol Res. 2014;2014: 621270. https://doi.org/ $10.1155 / 2014 / 621270$.

10. Espinosa G, Cervera R. Antiphospholipid syndrome: frequency, main causes and risk factors of mortality. Nat Rev Rheumatol. 2010;6(5):296-300. https://doi.org/10.1038/nrrheum.2010.47.

11. Nordestgaard BG, Langsted A. Lipoprotein (a) as a cause of cardiovascular disease: insights from epidemiology, genetics, and biology. J Lipid Res. 2016;57(11):1953-75. https://doi.org/10.1194/jlr. R071233.

12. Romero FI, Atsumi T, Tinahones FJ, Gómez-Zumaquero JM, Amengual O, Khamashta MA, Hughes GR. Autoantibodies against malondialdehydemodified lipoprotein(a) in antiphospholipid syndrome. Arthritis Rheum. 1999;42(12):2606-11. https://doi.org/10.1002/1529-0131(199912)42:12\% 3c2606::AID-ANR14\%3e3.0.CO;2-X.

13. Moraes-Silva IC, Rodrigues B, Coelho-Junior HJ, Feriani DJ, Irigoyen MC. Myocardial infarction and exercise training: evidence from basic science. Adv Exp Med Biol. 2017;999:139-53. https://doi.org/10. 1007/978-981-10-4307-9_9.

14. Smilowitz NR, Subramanyam P, Gianos E, Reynolds HR, Shah B, Sedlis SP. Treatment and outcomes of type 2 myocardial infarction and myocardial injury compared with type 1 myocardial infarction. Coron Artery Dis. 2018;29(1):46-52. https://doi.org/10. 1097/MCA.0000000000000545.

15. Amin MM, Ibrahim AM, Fahmy EM, Yassin AS, Abu-Elhassan SG, Abd-Elsadik A. Prognostic value of serum antiphospholipid antibodies in patients with ST-segment elevation myocardial infarction. Egypt J Immunol. 2018;25(1):143-51. 
16. Urbanus RT, Siegerink B, Roest M, Rosendaal FR, de Groot PG, Algra A. Antiphospholipid antibodies and risk of myocardial infarction and ischaemic

stroke in young women in the RATIO study: a casecontrol study. Lancet Neurol. 2009;8(11):998-1005. https://doi.org/10.1016/S1474-4422(09)70239-X. 